\title{
Breakthroughs in the Management of Hepatocellular Carcinoma: Celebrating 50 Years of the Liver Cancer Study Group of Japan
}

\author{
Masatoshi Kudo \\ Department of Gastroenterology and Hepatology, Kinki University School of Medicine, Osaka-Sayama, Japan
}

The 50th Liver Cancer Study Group of Japan (LCSGJ) Congress (Congress President: Prof. Masatoshi Kudo) was held in Kyoto, Japan, on June 5-6, 2014. The LCSGJ is an academic society which was founded in 1967 by Dr. Ichio Honjo, Professor of the Department of Surgery at Kyoto University. On March 7, 1949, while working at Kokura Memorial Hospital, Prof. Honjo (fig. 1), at the young age of 35 , became the first surgeon in the world to successfully perform an anatomical right lobectomy. Although the Japanese journal Shujutsu (Operation) published this breakthrough in 1950 [1], because it was not published in an English language journal until 1955 [2], there was a period in which articles cited the surgical technique performed by Lortat-Jacob [3] as the world's first anatomical right lobectomy. However, in the global history of liver surgery, Prof. Honjo is now well recognized as the pioneer who successfully completed the procedure first (fig. 2, 3) [4]. Prof. Honjo subsequently founded the LCSGJ and served as its first president in 1967. Largely due to the historical background, the LCSGJ maintained a head office at Kyoto University until 2008, when it moved to its current location, the Department of Gastroenterology and Hepatology at Kinki University (Head Office Representative: Prof. Masatoshi Kudo).

The theme of the 50th LCSGJ Congress was selected as 'Breakthroughs in the Management of Hepatocellular
Carcinoma', to celebrate the breakthrough made by Prof. Honjo as well as the numerous breakthroughs in treatments for liver cancer made in the intervening 50 years. The congress program was packed with special features:

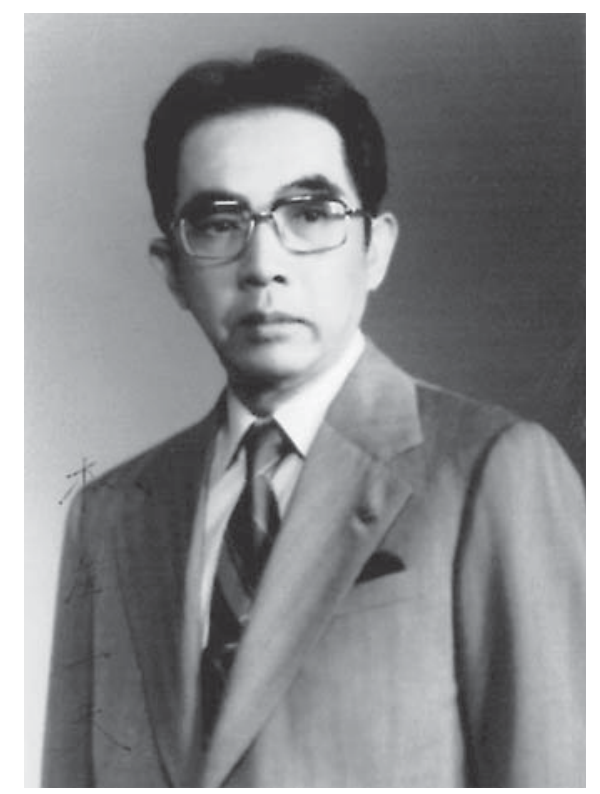

Fig. 1. Prof. Ichio Honjo (1913-1987), Professor Emeritus, Kyoto University School of Medicine (courtesy of Prof. Ryuji Mizumoto, Professor Emeritus, Mie University School of Medicine).

\section{KARGER}

E-Mail karger@karger.com www.karger.com/ocl
(C) 2014 S. Karger AG, Basel

0030-2414/14/0877-0001\$39.50/0
Prof. Masatoshi Kudo

Department of Gastroenterology and Hepatology Kinki University School of Medicine

377-2 Ohno-Higashi, Osaka-Sayama, Osaka 589-8511 (Japan)

E-Mail m-kudo@med.kindai.ac.jp 


\section{History of Liver Surgery \\ James H. Foster, MD}

Honjo and $\mathrm{Araki}^{+3}$ may have performed an anatomic right lobectomy for metastatic carcinoma in Japan in 1949, but their case was not reported until after that of the French surgeons. In 1952, Lortat-Jacob and Robert did indeed perform what may have been an extended right lobectomy in a 42-year-old woman with multiple metastatic lesions. ${ }^{35}$ The patient apparently recovered from the operation, but there was no late follow-up information available about her future course. In the same year, the Quattlebaums (father and son), of Augus-

Fig. 2. Extracted sentences from an article published in Archives of Surgery [4]. Dr. Foster clearly states that Prof. Honjo performed the world's first anatomic right hepatectomy in Japan in 1949. two special lectures, one education lecture, one roundtable-style commemorative session on the LCSGJ's history, three symposia, seven panel discussions, nine workshops, six video sessions, six difficult clinical case study sessions, and two consensus meetings (table 1).

In Symposium 1 entitled 'Basic Aspect, Diagnosis, Treatment, and Prognosis of Early Hepatocellular Carcinoma', discussions were held on next-generation sequencing analysis and diagnostic imaging for early hepatocellular carcinoma (HCC) [5], diagnosis by Sonazoidenhanced ultrasonography and gadolinium ethoxybenzyl diethylenetriamine pentaacetic acid MRI, issues associated with pathological diagnosis, and the timing of treatment. Symposium 2 provided a forum for discussion in line with the session 'Treatment Strategies for Advanced and Large Liver Cancer' and particularly the attempts to

\section{SOLID LIVER TUMORS}

by

James H. Foster, M.D.

Director, Department of Surgery Hartford, Connecticut

and

Martin M. Berman, M.D.

Department of Patholog
Hartord Hospital

Hartford, Connecticu

Assistant Professor of Pathology

Health Center

Farmington, Connecticut

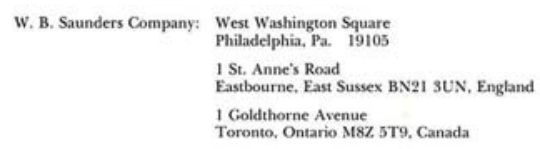

Eastbourne, East Sussex BN21 3UN, England

Toronto, Ontario M8Z 5T9, Canada

a W. B. Saunders Company, Philadelphia, London, Toronto, 1977

Volume XXII in the Series MAJOR PROBLEMS IN CLINICAL SURGERY

PAUL A. EBERT, M.D.

Consulting Editor

Solid Liver Tumors

ISBN $\quad 0.7216-3824-4$

(C) 1977 by W. B. Saunders Company. Copyright under the International Copyrighs Union. All rights reserved. This book is protected by copyright. No part of it may be reproduced, stored in a electronic. mechanical, photocopying, recording, or otherwise, without written permission
from the publisher. Made in the United States of America. Press of W, B. Saunders Company. Library of Congress catalog card number 76-028938.

Last digit is the print number:

Fig. 3. Extracts from the textbook by Dr. Foster and Dr. Berman (courtesy of Prof. Ryuji Mizumoto) [30]. a Cover picture of Solid Liver Tumors. 
combine surgery and chemotherapy to treat vascular invasion, bile duct invasion, and large liver cancer. In Symposium 3, the heterogeneity of intermediate stage HCC, the attempt to subgroup heterogeneous intermediate stage HCC, the definition of transarterial chemoembolization (TACE) failure/refractoriness, and the treatment options for TACE failure/refractoriness cases were discussed under the title 'Treatment Strategies, Efficacy, and Prognosis of Intermediate Stage Liver Cancer'.

Highlights in the History of Liver TUmors and Their Resection 21

In 1910 Professor Walther Wendell resected a double-fist-sized liver cell "adenoma" from a 44-year-old woman after deliberately tying the right branch of the hepatic artery and the right hepatic duct at the liver hilum. He did not attempt ligation of the right portal vein because he worried about thrombosis of the vein extending into the remaining left branch. The patient did well, and was alive and free of apparent disease eight months later ${ }^{89}$

In the modern era, credit for the first anatomic lobectomy based on vascular anatomy with preliminary hilar ligation is usually given to Lortat-Jacob and Robert (1952),4 but McBride ${ }^{52}$ credits Caprio (1931) with the first anatomic lobectomy. ${ }^{5}$ Unfortunately, Caprio's paper was not available for confirmation, and the condition for which his operation was done is not known. Pettinari is said to have done an anatomic lobectomy in $1940{ }^{62}$ perhaps for hypertrophic tuberculosis (see Dagradi and Brearley, case 5). ${ }^{11}$ Donovan and Santulli in 1944 removed the lateral segment of the left lobe of a 6-year-old boy for a mesenchymoma after tying the branches of the left hepatic duct, portal vein, and left hepatic artery in the interlobar fissure. ${ }^{12}$ Hershey performed a sim. ilar operation in Wheeling, West Virginia in $1945 .^{94}$ Fineburg et al. (1956) tabulated all the reported cases of right hepatic lobectomy, and it would appear that that of Lortat-Jacob and Robert may have been the fifth done with primary hilar ligation, rather than the first. ${ }^{17}$ Fineburg credits Honjo and Araki with the resection of a right lobe for metastatic colon carcinoma in 1949 , but when these Japanese authors reported their case in 1955 they claimed no priority over Lortat-Jacob, whom they cite. ${ }^{29}$ However, the Japanese report was submitted for publication in 1953 and contained more than a year's follow-up information on their patient, so their operation may well have preceded that of-the-French.

Questions of priority aside, several major hepatic lobectomies for solid tumor were done in the first half of the 1950s, and the reports thereof stimulated a widespread acceptance of the procedures. LortatJacob and Robert operated on a 42-year-old woman with multiple metastatic lesions involving her right lobe and the medial segment of the left lobe of the liver. Through a combined thoracoabdominal incision, a "controlled" hepatectomy was done after primary hilar ligation. The postoperative course was reasonably benign, but there was no late follow-up on this patient. ${ }^{4}$ Pack and Baker reported total right hepatic lobectomy with primary hilar ligation for a female patient with cancer of the gallbladder in 1953. Unfortunately, the patient died with diffuse metastases in March 1954.61 In Augusta, Georgia, Julian Quattlebaum resected a primary hepatoma from a 65 -year-old woman in 1952 by right lobectomy after primary hilar ligation. The patient died 19 months later with recurrent disease. Quattlebaum had performed two other right lobectomies for angiomas in the 13 months prior to the

b hepatoma case, but these were done by guillotine transection..$^{65}$

Fig. 3. Extracts from the textbook by Dr. Foster and Dr. Berman (courtesy of Prof. Ryuji Mizumoto) [30]. b Exracted sentences from the textbook. Dr. Foster clearly states 'Honjo and Araki's operation may well have preceeded that of the French'.

Breakthroughs in the Management of HCC
Latest diagnostic imaging modalities [6-9] were introduced in Panel Discussion 1, entitled 'Innovation in Diagnostic Imaging for Liver Cancer', while basic research approaches using, for example, oncogenes and methylation were discussed in Panel Discussion 2 on 'Future Prospects in Liver Cancer Treatment Based on Genome/Epigenome Data'. In Panel Discussion 3 on 'Future Perspectives on Molecular Targeted Therapy for Liver Cancer', discussions centered around the application of molecular targeted therapy, for which sorafenib is currently the only approved drug, the combination of sorafenib with conventional locoregional treatments [10-18], and other emerging targeted agents. In Panel Discussion 4, entitled 'Current Situation and Ongoing Challenges in Liver Transplantation for $\mathrm{Pa}$ tients with Hepatocellular Carcinoma', current issues associated with liver transplantation were addressed, while in Panel Discussion 5, 'Diagnosis and Pathology of Cystic Liver Tumor', new concepts such as intraductal papillary neoplasm of the intrahepatic bile duct and mucinous cystic neoplasm were reviewed. In Panel Discussion 6, pathological features were debated under the theme 'Pathology and Diagnosis of Combined Hepatocellular and Cholangiocellular Carcinoma'. Lastly, in Panel Discussion 7 on 'Various Issues Associated with Hepatocellular Adenoma and Focal Nodular Hyperplasia', the subgroups of hepatocellular adenomas, which have been attracting attention in recent years, and the imaging findings of focal nodular hyperplasia were reviewed.

Nine workshops were held at the congress. In Workshop 1 on 'Treatment for Child-Pugh C Liver Cancer', it was verified that locoregional therapy confers survival benefit to a specific subgroup of liver cancer patients with Child-Pugh $\mathrm{C}$ liver function. The discussions in Workshop 2 entitled 'Treatment Strategies for Recurrent Liver Cancer after Liver Transplantation' focused on recurrence, the most serious problem caused by liver transplantation [19-23]. In Workshop 3, the mechanism of and clinical approach to nonalcoholic steatohepatitis (NASH)-induced liver cancer were discussed under the theme of 'Basic and Clinical Aspects of NASHInduced Liver Cancer: Including Diagnostic Criteria' [24]. Workshop 4 on 'Long-term Survival Cases $(\geq 3$ Years) of Advanced Hepatocellular Carcinoma after the Initiation of Molecular Targeted Therapy' focused on the characteristics of long-term survival cases after treatment with sorafenib. In Workshop 5 on 'Treatment Strategy for Intrahepatic Cholangiocarcinoma', surgical outcome of intrahepatic cholangiocarcinoma and the 
Table 1. The 50th LCSGJ Congress: Special Programs

\begin{tabular}{|c|c|}
\hline Special Lecture & $\begin{array}{l}1 \text { Full Lifecycle Aquaculture of Bluefin Tuna: 32-Year Trajectory, by Prof. Hidemi Kumai } \\
2 \text { Management of Liver Cancer - Why We Do What We Do in Europe, by Prof. Markus Peck-Radosavljevic }\end{array}$ \\
\hline Memorial Roundtable & Looking Back on the 50-Year History of the Liver Cancer Study Group of Japan \\
\hline Symposium & $\begin{array}{l}1 \text { Basic Aspect, Diagnosis, Treatment, and Prognosis of Early Hepatocellular Carcinoma } \\
2 \text { Treatment Strategies for Advanced and Large Liver Cancer } \\
3 \text { Treatment Strategies, Efficacy, and Prognosis of Intermediate Stage Liver Cancer }\end{array}$ \\
\hline Workshop & $\begin{array}{ll}1 & \text { Treatment for Child-Pugh C Liver Cancer } \\
2 & \text { Treatment Strategies for Recurrent Liver Cancer after Liver Transplantation } \\
3 & \text { Basic and Clinical Aspects of NASH-Induced Liver Cancer: Including Diagnostic Criteria } \\
4 & \text { Long-term Survival Cases ( } \geq 3 \text { Years) of Advanced Hepatocellular Carcinoma after the Initiation of Molecular Targeted } \\
& \text { Therapy } \\
5 & \text { Treatment Strategy for Intrahepatic Cholangiocarcinoma } \\
6 & \text { Radiation Therapy for Hepatocellular Carcinoma } \\
7 & \text { Treatment Strategy for Colorectal Liver Metastasis } \\
8 & \text { Current Situation and Recent Advances in RFA } \\
9 & \text { New Developments in TACE: How to Apply Beads TACE and cTACE Differently }\end{array}$ \\
\hline Video Session & $\begin{array}{l}1 \text { State-of-the-Art Contrast-Enhanced Ultrasonography } \\
2 \text { Advances in Simulation Imaging (Including 3- and 4-Dimensional Imaging) of Liver Cancer Treatment } \\
3 \text { Resection of Hepatocellular Carcinoma: Highly Technical Surgical Procedures } \\
4 \text { Technological Advances in TACE } \\
5 \text { The Frontline of Laparoscopic Therapy for Hepatocellular Carcinoma } \\
6 \text { Technological Advances in RFA Therapy (Including Fusion Imaging) }\end{array}$ \\
\hline
\end{tabular}

characteristics of recurrence were discussed with a focus on hepatectomy. Workshop 6 focused on 'Radiation Therapy for Hepatocellular Carcinoma', and the participants discussed treatment directed solely at vascular invasion or the main lesion of HCC itself and proton and particle therapy $[25,26]$. In addition, under the theme 'Treatment Strategy for Colorectal Liver Metastasis' in Workshop 7, the participants engaged in an active discussion of systemic chemotherapy, surgical approaches, microwave coagulation therapy, radiofrequency ablation (RFA), or particle therapy as locoreginal treatments.
The main focus in Workshop 8, entitled 'Current Situation and Recent Advances in RFA' was bipolar RFA, and new RFA techniques including fusion image-guided RFA were also discussed [27]. Lastly, in Workshop 9 entitled 'New Developments in TACE: How to Apply Beads TACE and cTACE Differently', the participants discussed the differential application of conventional TACE [28] and beads (microsphere) TACE, the latter of which was introduced to Japan in January 2014.

In Video Session 1 entitled 'State-of-the-Art Contrast-Enhanced Ultrasonography', the significance of 
this imaging modality for treatment guidance was discussed, as was its value in fusion imaging, in pretreatment diagnosis of malignant grade, in establishing treatment indications, and in selecting an appropriate treatment approach. Video Session 2 featured 'Advances in Simulation Imaging (Including 3- and 4-Dimensional Imaging) of Liver Cancer Treatment' and their applications. Extremely difficult surgical methods in liver resection were introduced and debated in Video Session 3 entitled 'Resection of Hepatocellular Carcinoma: Highly Technical Surgical Procedures', while 'Technological Advances in TACE' (e.g., beads TACE, balloon occluded-TACE, and FlightPlan) was the theme in Video Session 4. Video Session 5 on 'The Frontline of Laparoscopic Therapy for Hepatocellular Carcinoma' covered laparoscope-assisted treatment and robotic surgery with the da Vinci surgical system. Lastly, Video Session 6 entitled 'Technological Advances in RFA Therapy (Including Fusion Imaging)' introduced various recent advances in RFA. The six Difficult Clinical Case Study Sessions comprised three sessions on diagnosis and three on treatment, all of which were conducted with a moderator who was either a hepatologist, a surgeon, a pathologist, or a radiologist. In each session, a difficult case in terms of diagnosis or treatment was introduced, and the diagnosis or treatment approach in question was actively debated.

As a special program, a roundtable-style presentation was held under the title 'Looking Back on the 50-Year History of the Liver Cancer Study Group of Japan', and eight professors who had led the LCSGJ through the last 50 years introduced the history of the LCSGJ to the younger generation of LCSGJ members. This special program also provided a great opportunity to think about how we should develop the LCSGJ over the next 50 years.

In the Education Lecture, Prof. Kazuto Nishio gave a speech about 'Biomarkers in Molecular Targeted Therapy for Liver Cancer', which provided up-to-date information on biomarkers. In addition, Prof. Hidemi Kumai and Prof. Markus Peck presented highly relevant Special Lectures on 'Full Lifecycle Aquaculture of Bluefin Tuna: 32Year Trajectory' and 'Management of Liver Cancer Why We Do What We Do in Europe', respectively.

Two consensus meetings organized by the LCSGJ turned out to be the highlight of the 50th Congress. In Consensus Meeting 1 on 'A Diagnostic Algorithm for Liver Cancer', liver cancer experts used a voting system to decide on an updated version of the consensus-based diagnostic algorithm proposed by the Japan Society of Hepatology (JSH) [29]. In Consensus Meeting 2 on 'Updating the JSH Definition of TACE Failure/Refractoriness', experts decided to revise the definition [29].

The 50th LCSGJ Congress drew over 1,200 attendees over 2 days and was a great success. In this supplementary issue of Oncology, some of the articles discuss the sessions held at the congress, making it an extremely valuable special issue for readers specializing in liver cancer.

\section{Disclosure Statement}

The author declares that no financial or other conflicts of interest exist in relation to the content of this article.

\section{References}

1 Honjo I: Total resection of the right lobe of the liver (in Japanese). Shujutsu 1950;4:345-349.

$\checkmark 2$ Honjo I, Araki C: Total resection of the right lobe of the liver; report of a successful case. J Int Coll Surg 1955;23:23-28.

-3 Lortat-Jacob JL, Robert HG: Well defined technic for right hepatectomy. Presse Med 1952;60:549-551.

4 Foster JH: History of liver surgery. Arch Surg 1991;126:381-387.

5 Kudo M: Early hepatocellular carcinoma: definition and diagnosis. Liver Cancer 2013;2: $69-72$.

-6 Murakami T, Tsurusaki M: Hypervascular benign and malignant liver tumors that require differentiation from hepatocellular carcinoma: key points of imaging diagnosis. Liver Cancer 2014;3:85-96.

Breakthroughs in the Management of HCC
7 Lee JM, Yoon JH, Joo I, Woo HS: Recent advances in CT and MR imaging for evaluation of hepatocellular carcinoma. Liver Cancer 2012;1:22-40

8 Joo I, Choi BI: New paradigm for management of hepatocellular carcinoma by imaging. Liver Cancer 2012;1:94-109.

-9 Salvatore V, Bolondi L: Clinical impact of ultrasound-related techniques on the diagnosis of focal liver lesions. Liver Cancer 2012;1: 238-246.

10 Kudo M: Treatment of advanced hepatocellular carcinoma with emphasis on hepatic arterial infusion chemotherapy and molecular targeted therapy. Liver Cancer 2012;1:62-70.

11 Kudo M: Why does every hepatocellular carcinoma clinical trial using molecular targeted agents fail? Liver Cancer 2012;1:59-60.
12 Hsu C, Po Ching L, Morita S, Hu FC, Cheng AL: Perspectives on the design of clinical trials combining transarterial chemoembolization and molecular targeted therapy. Liver Cancer 2012;1:168-176.

13 Liapi E, Geschwind JF: Combination of local transcatheter arterial chemoembolization and systemic anti-angiogenic therapy for unresectable hepatocellular carcinoma. Liver Cancer 2012;1:201-215.

14 Finn RS: Current and future treatment strategies for patients with advanced hepatocellular carcinoma: role of mTOR inhibition. Liver Cancer 2012;1:247-256.

15 Shindoh J, Kaseb A, Vauthey JN: Surgical strategy for liver cancers in the era of effective chemotherapy. Liver Cancer 2013;2:47-54. 
16 Shao YY, Hsu CH, Cheng AL: Predictive biomarkers of antiangiogenic therapy for advanced hepatocellular carcinoma: where are we? Liver Cancer 2013;2:93-107.

17 Kim HY, Park JW: Clinical trials of combined molecular targeted therapy and locoregional therapy in hepatocellular carcinoma: past, present, and future. Liver Cancer 2014;3:9-17.

18 Peck-Radosavljevic M: Drug therapy for advanced-stage liver cancer. Liver Cancer 2014; 3:125-131.

19 Belghiti J, Fuks D: Liver resection and transplantation in hepatocellular carcinoma. Liver Cancer 2012;1:71-82.

20 Lee Cheah Y, K H Chow P: Liver transplantation for hepatocellular carcinoma: an appraisal of current controversies. Liver Cancer 2012; $1: 183-189$.
21 Chan SC, Sharr WW, Chan AC, Chok KS, Lo CM: Rescue living-donor liver transplantation for liver failure following hepatectomy for hepatocellular carcinoma. Liver Cancer 2013;2:332-337.

22 Chan SC: Liver transplantation for hepatocellular carcinoma. Liver Cancer 2013;2:338344.

23 Akamatsu N, Sugawara Y, Kokudo N: Living donor liver transplantation for patients with hepatocellular carcinoma. Liver Cancer 2014; 3:108-118

24 Kim DY, Han KH: Epidemiology and surveillance of hepatocellular carcinoma. Liver Cancer 2012;1:2-14.

25 Jihye C, Seong J: Application of radiotherapeutic strategies in the BCLC-defined stages of hepatocellular carcinoma. Liver Cancer 2012;1:216-225.
6 Lee DS, Seong J: Radiotherapeutic options for hepatocellular carcinoma with portal vein tumor thrombosis. Liver Cancer 2014;3:18-30.

27 Lin SM: Local ablation for hepatocellular carcinoma in Taiwan. Liver Cancer 2013;2:7383.

28 Lencioni R: Chemoembolization in patients with hepatocellular carcinoma. Liver Cancer 2012;1:41-50.

29 Kudo M, Matsui O, Izumi N, et al: JSH consensus-based clinical practice guideline for the management of hepatocellular carcinoma: 2014 update by the Liver Cancer Study Group of Japan. Liver Cancer 2014;3:458468.

30 Foster JM, Berman MM: Solid Liver Tumors. Philadelphia, WB Saunders, 1977. 